\title{
Design and Reality: Continuous-flow Accelerator Mass Spectrometry (CFAMS)
}

\author{
Karl F. von Reden ${ }^{1}$, Mark L. Roberts ${ }^{1}$, Cameron P. McIntyre ${ }^{1}$, Joshua R. Burton ${ }^{1}$ \\ ${ }^{1}$ National Ocean Sciences AMS Facility, Department of Geology and Geophysics, \\ Woods Hole Oceanographic Institution, Woods Hole, MA 02543, USA
}

\begin{abstract}
In 2007 we published [1] the design of a novel accelerator mass spectrometry (AMS) system capable of analyzing gaseous samples injected continuously into a microwave plasma gas ion source. Obvious advantages of such a system are drastically reduced processing times and avoidance of potentially contaminating chemical preparation steps. Another paper in these proceedings will present the progress with the development of the microwave gas ion source that has since been built and tested at the National Ocean Sciences AMS Facility in Woods Hole [2]. In this paper we will review the original design and present updates, reflecting our recent encouraging experience with the system. A simple summary: large acceptance ion beam optics design is beneficial to accelerator mass spectrometry in general, but essential to AMS with plasma gas ion sources.

PACS: 41.85; 07.77.Ka; 07.75.+h

Keywords: Ion optics; Computer modeling; Microwave ion source; Continuous-flow AMS
\end{abstract}

\section{Introduction}

A ${ }^{14} \mathrm{C}$ AMS system based on a true gas ion source is highly desirable as it avoids the major chemical preparation step of reducing $\mathrm{CO}_{2}$ to elemental carbon required in a negative ion sputter source. Conventional AMS makes use of the fact that the dominant isotope of mass 14, nitrogen, does not form negative ions - contrary to carbon, allowing the analysis of ${ }^{14} \mathrm{C}$ with very high abundance sensitivity. To date it has not been shown that negative carbon ions can be efficiently extracted from the plasma of a gas ion source. This makes it necessary to couple such an ion source to a charge exchange canal $(\mathrm{CxC})$, an approach we have described elsewhere [3-6]. While the total negative ion efficiency of such an assembly is at least 1-2 orders of magnitude lower 
than that of a modern sputter ion source, the very large positive currents extractable from a microwave plasma allow the analyzable negative currents to be comparable to an average sputter source, if sample size is not the limiting factor. The intrinsically high emittance of a plasma gas ion source, caused mainly by the $\sim 5 \mathrm{~mm}$ diameter orifice of the plasma chamber, is a challenge to the AMS system design. That makes the effective diameter of the beam origin 2-3 mm, at least doubling the emittance in comparison to a high current sputter source. In the original design [1] we attempted to address the beam size problem by introducing very large gaps in all beam optics devices and making both the low-energy and the high-energy analyzers achromatic to overcome the energy spreads from $\mathrm{CxC}$ and stripper canal. In addition, we designed the $\mathrm{CxC}$ to be biased at $-30 \mathrm{kV}$ (doubling as extraction potential for the source at ground), thereby raising the beam energy to $\sim 60 \mathrm{keV}$, a better match to the $500 \mathrm{kV}$ Pelletron. The main limitation of the system was identified: the size of the stripper canal. In more than three years of operation it has been well established that the high-energy beam optics of the new system is more than adequate to transport any ion beams emerging from the stripper canal without loss. Therefore we will discuss here only the implementation of the low-energy section of the CFAMS design.

\section{Microwave Ion Source}

The principal design of the plasma ion source has not changed from the original form [7] and the prototype described in refs. $4-6$. However, the decision to integrate the extraction and suppression electrodes with the biased CxC, thereby mechanically decoupling them from the plasma chamber, proved to be a very difficult problem during operation. Reproducible XYZ alignment of the $\mathrm{CxC}$, keeping the apertures plan parallel and on axis was not possible with sufficient accuracy. Other issues, like voltage stability and temperature control of the CxC added to the operational difficulties, eventually causing us to temporarily abandon the $\mathrm{CxC}$ biasing 
idea, at least for the present implementation. This reflects a major change in the design parameters described in [1]: beam energy, emittance, and energy spread. Nothing can be done about the beam energy, and energy spread is still compensated by the achromatic system elements. However, we redoubled our effort to better understand the emittance of the source. Our original calculations with code PBGUNS [8] assumed a Maxwellian velocity distribution of the plasma ions, resulting in a beam divergence that was in rough agreement with measurements on a test stand with two beam profile monitors [9]. Our latest experimental setup allows us to view the extracted beam through a side window (CxC removed), giving us a first-order assessment of the positive ion beam shape under varying extraction conditions. A stainless steel plate (now replaced with a tantalum plate) at the exit of the $\mathrm{CxC}$ box with a $12 \mathrm{~mm}$ diameter aperture centered on the beam axis helps us with the alignment of the extraction triode during operation. This plate is located $300 \mathrm{~mm}$ downstream from the plasma chamber orifice. Figure 1 shows a 5 mm diameter burn mark on the plate from a short exposure to the extracted beam from slightly misaligned extraction apertures. Calculations with PBGUNS can reproduce that beam shape only with a non-Maxwellian approach, but introducing some angular spread to the extracted ions. Figure 2 shows the results of that calculation, suggesting that over $70 \%$ of the beam emerges in a cone of less than 5 mrad half angle. The calculated current density distribution (fig. 2d) closely resembles the pattern created on the steel plate. Introducing the $\mathrm{CxC}$ adds $+/-325 \mathrm{eV}$ of energy spread. In the following sections we will compare beam optics calculations based on these beam parameters with observed transmission results.

\section{Injector Modeling}

The beam emerging from the CxC is composed of $\sim 20 \%$ positive, $~ 20 \%$ negative and $\sim 60 \%$ neutral particles (see figure 2 in ref. 5). In our present setup we cannot directly measure the 
extracted current, but estimate it to be several mA (including back flowing electrons), from power supply readings. While we are able to operate the Einzel lens under these conditions, the electrostatic analyzer (ESA) breaks down under direct exposure to this beam. We therefore introduced a $7.5^{\circ}$ electrostatic deflection near the theoretical object point of the ESA. There is no significant effect on the beam shape from this device and the ESA can now be operated without breakdowns. The original design of the injector was based on a $60 \mathrm{keV}$ beam. The trajectory calculations in this paper were performed with the same approach that was used in [1]: finiteelement modeling with $5 \mathrm{~mm}$ mesh tetrahedron size in field regions [10]. Figure 3 shows the result of calculating the trajectories for ${ }^{12} \mathrm{C}$ and ${ }^{13} \mathrm{C}$ negative ion beams with $\pm 325 \mathrm{eV}$ spread around $34.5 \mathrm{keV}$ kinetic energy, $5 \mathrm{~mm}$ diameter at the exit of the $\mathrm{CxC}$, and 6 mrad divergence half angle. A few eV of energy spread in the plasma ion source were neglected. The calculations use the operational parameters established by tuning the system for optimal transmission, listed in table 1 in comparison with parameters from the original design. The beam emerging from the Einzel lens is nearly parallel with a waist $\sim 250 \mathrm{~mm}$ downstream from the theoretical object point of the ESA. The horizontal and vertical waists after the ESA are substantially upstream of its theoretical image point, causing the beam to be diverging at this location, where in our setup a beam profile monitor (BPM) is installed. Under optimal tuning conditions the lateral size of the beam at this point is over $20 \mathrm{~mm}$ (including all other ion beam components with charge state -1). BG1 indicates the electrostatic bouncer field gap on the entrance of the biased dipole magnet chamber. This device is in our installation just a standard Ceramaseal isolator with $23 \mathrm{~mm}$ gap, $100 \mathrm{~mm}$ diameter. The sequential injection scheme we are using sets the bias for ${ }^{13} \mathrm{C}$ at $+6 \mathrm{kV}$. That requires a bias for ${ }^{12} \mathrm{C}$ of $+9.375 \mathrm{kV}$. Insert (a) in figure 3 shows only a small focusing effect of the gap on the two ion species. The detail is scaled and horizontally stretched by a factor of 10 to make individual rays visible. Black and white trajectories demark ${ }^{12} \mathrm{C}$ and ${ }^{13} \mathrm{C}$, respectively. 
With otherwise identical beam parameters, the higher bias leads to slightly stronger focusing for

${ }^{12} \mathrm{C}$ at this point. Due to the upstream shift of the object point of the dipole magnet the horizontal and vertical waists of the beam also are shifted upstream and fall short of the theoretical image point (BG2). There is also a slight focal plane rotation, shifting the focus of the ${ }^{12} \mathrm{C}$ beam upstream of the ${ }^{13} \mathrm{C}$ component by $\sim 24 \mathrm{~mm}$ (not shown in the figure). There is no significant difference in the focusing effect on ${ }^{12} \mathrm{C}$ and ${ }^{13} \mathrm{C}$ of the $3.2 \mathrm{~mm}$ field gap (BG2). Inserts (b) and (c) in figure 3 show details of the $0^{\circ}$ line through the low-energy end of the accelerator. Again the diagrams are compressed in beam direction to improve the visibility of trajectories. It is clear that transmission problems are mainly caused by the size of the beam in horizontal direction. While the achromatic configuration of the injector reasonably well overcomes the energy spread of the beam it cannot avoid an accompanying angular spread. The larger divergence in the horizontal plane leads to losses in the outer part of the beam. This affects the lighter ${ }^{12} \mathrm{C}$ particles more than ${ }^{13} \mathrm{C}$ because the ${ }^{12} \mathrm{C}$ waist is slightly upstream from the ${ }^{13} \mathrm{C}$ waist (indicated in insert (c)).

Neglecting other effects (like angular straggling in the stripper canal) it is clear that our main problem is poor energy matching of the injected ion beam to the accelerator, causing a “premature” focus ahead of the stripper canal. Until we can return to the original design, other venues have to be explored to improve the ion optics in our system.

\section{Discussion}

In our AMS operation with the microwave gas ion source we presently observe ${ }^{12} \mathrm{C}^{-}$currents at the entrance of the accelerator of about $140 \mu \mathrm{A}$ and about $40 \mu \mathrm{A}$ of ${ }^{12} \mathrm{C}^{+}$after the high energy analyzing magnet, equaling $29 \%$ transmission. That is $70 \%$ of the transmission obtained with a sputter source beam (42\% charge exchange yield at $525 \mathrm{kV}$ terminal voltage, 25 mTorr argon pressure at canal center). However, there is a considerable difference in losses for the three 
isotopes of carbon resulting in fractionation, i.e. the expected isotope ratios for known sample materials are not reproduced by the analyzed currents and count rates. An offset of $+12 \%$ is seen for ${ }^{13} \mathrm{C} /{ }^{12} \mathrm{C}$ and $+24 \%$ for ${ }^{14} \mathrm{C} /{ }^{12} \mathrm{C}$, resulting in ${ }^{14} \mathrm{C}$ count rates for modern samples approaching 400/s. The conventional AMS correction for $\delta^{13} \mathrm{C}$ brings the results into good agreement with expected results for all known standards. While the size of the offsets is difficult to predict in modeling calculations, the results presented in section 3 qualitatively explain the observations. It is important to note that changes in tuning parameters, especially the magnetic solenoid field in the ion source do not significantly affect the fractionation, indicating that mass dependent losses in the stripper canal must be the main cause for the offsets.

There are several pathways to improving our system design. Ultimately, it is still our goal to go back to the biased $\mathrm{CxC}$ method of the original design [1]. However, even there it was clear that the horizontal beam size was too large for the existing stripper canal. As the trajectory calculations in section 3 showed, it only takes a moderate increase in the exit size of the stripper canal to allow the cores of all three isotope ion beams to pass freely. This would certainly reduce the observed fractionation but not eliminate it completely. Another approach might be to replace the stripper canal with a foil stripping device. Earlier attempts to harness carbon nanotube meshes for electron stripping in tandem accelerators [11] showed that it is possible to make freestanding sheets of less than 100 atomic layers with superior mechanical properties. Their endurance under ion beam exposure was comparable to but not better than that of conventional carbon foils. Now there are major developments in the field of nanotechnology, which may soon allow the industrial production of macroscopic graphene sheets (single layer honeycomb structure) of unprecedented mechanical properties [12]. It is conceivable to have spools of graphene ribbon installed in the terminal of a tandem with several years of stripping capacity in one cassette. 
We believe that the other known shortcomings of the present design, size and location of the first bouncer gap (BG1), focal plane rotation of the injector magnet, are much less important than the stripper canal problem, but will address them as well over time. Further reduction of the emittance of the gas ion source will remain another field of study for us.

\section{Conclusions}

We implemented the design of a continuous-gas-flow AMS system at the Woods Hole Oceanographic Institution's NOSAMS Facility, based on a microwave gas ion source. Even though technical difficulties forced us to temporarily abandon a challenging design detail - a biased charge exchange canal - we were able to demonstrate very promising results with the new system, highlighted in another paper in these proceedings [2]. We believe that the ability to analyze samples from a flowing stream of gas with minimal sample-to-sample memory (as it is common place in stable isotope mass spectrometry) will open new avenues in ${ }^{14} \mathrm{C}$ AMS. Design improvements, as addressed in this paper and further development of well-matched gas delivery methods for the new AMS system will be the next phase of activities at NOSAMS.

\section{Acknowledgment}

This work is supported by a Cooperative Agreement (OCE-0753487) with the U.S. National Science Foundation.

\section{References}

[1] BX Han, KF von Reden, ML Roberts, RJ Schneider, JM Hayes, WJ Jenkins. Electromagnetic field modeling and ion optics calculations for a continuous-flow AMS system, Nuclear Instruments and Methods in Physics Research Section B: Beam Interactions with Materials and Atoms. 259 (2007) 111-117. 
[2] M Roberts, KF von Reden, CP McIntyre, JR Burton. Progress with a gas-accepting ion source for Accelerator Mass Spectrometry. These Proceedings.

[3] RJ Schneider, KF von Reden, JSC Wills, WT Diamond, R Lewis, G Savard, et al. Hold-up and memory effect for carbon in a compact microwave ion source, Nuclear Instruments and Methods in Physics Research Section B: Beam Interactions with Materials and Atoms. 123 (1997) 546-549.

[4] RJ Schneider, KF von Reden, JM Hayes, JSC Wills, WGE Kern, S- Kim. Tests of positive ion beams from a microwave ion source for AMS, Nuclear Instruments and Methods in Physics Research Section B: Beam Interactions with Materials and Atoms. 172 (2000) 252-256.

[5] S Kim, RJ Schneider, KF von Reden, JM Hayes, JSC Wills. Test of negative ion beams from a microwave ion source with a charge exchange canal for accelerator mass spectrometry applications, Rev.Sci.Instrum. 73 (2002) 846-848.

[6] ML Roberts, RJ Schneider, KF von Reden, JSC Wills, BX Han, JM Hayes, et al. Progress on a gas-accepting ion source for continuous-flow accelerator mass spectrometry, Nuclear Instruments and Methods in Physics Research Section B: Beam Interactions with Materials and Atoms. 259 (2007) 83-87.

[7] JSC Wills, RA Lewis, J Diserens, H Schmeing, T Taylor. A compact high-current microwave-driven ion source, Rev.Sci.Instrum. 69 (1998) 65-68.

[8] JE Boers, PBGUNS, 5.04 (2001). WWW.FAR-TECH.COM.

[9] K von Reden, M Roberts, B Han, R Schneider, J Wills. Searching For A Suitable Gas Ion Source For [sup 14]C Accelerator Mass Spectrometry, AIP Conf.Proc. 925 (2007) 341-345.

[10] Infolytica Corporation, ElecNet \& MagNet, v.7 (2010). WWW.INFOLYTICA.COM.

[11] K von Reden, M Zhang, M Meigs, E Sichel, S Fang, RH Baughman. Carbon nanotube foils for electron stripping in tandem accelerators, Nuclear Instruments and Methods in Physics Research Section B: Beam Interactions with Materials and Atoms. 261 (2007) 44-48.

[12] YP Chen, Q Yu. Nanomaterials: Graphene rolls off the press, Nat Nano. 5 (2010) 559-560. 
Table 1: Tuning parameters in comparison with original design parameters

\begin{tabular}{|c|c|c|}
\hline & Original Design & Present \\
\hline Source $(\mathrm{kV})$ & 0 & 34.5 \\
\hline Suppression $(\mathrm{kV})$ & -31.5 & -3.5 \\
\hline CxC $(\mathrm{kV})$ & -30 & 0 \\
\hline Einzel Lens $(\mathrm{kV})$ & -40 & -19 \\
\hline $7.5^{\circ}$ Deflector $(\mathrm{kV})$ & N/A & -5 \\
\hline ESA $(\mathrm{kV})$ & 28.6 & 16.6 \\
\hline
\end{tabular}

\section{Figure Captions}

Fig. 1: $5 \mathrm{~mm}$ diameter burn mark created by the ion beam on a stainless steel aperture plate 300 $\mathrm{mm}$ downstream from the plasma aperture. Notice the central spot of $<1 \mathrm{~mm}$ diameter, reflecting the shape of the calculated current density in figure 2d.

Fig. 2: Calculations with code PBGUNS 5.04. (a) Trajectories of $\sim 4000$ particles (every $32^{\text {nd }}$ drawn) with five ion species considered: ${ }^{12} \mathrm{C}^{+},{ }^{16} \mathrm{O}^{+},{ }^{12} \mathrm{C}^{16} \mathrm{O}^{+},{ }^{16} \mathrm{O}^{+},{ }^{12} \mathrm{C}^{16} \mathrm{O}^{+}$(as measured in ref.2). The solenoid B field measured in our setup is indicated at the top. (b,c) Emittance plots at $19 \mathrm{~mm}$ and $300 \mathrm{~mm}$ distance from the plasma aperture. (d) Current density distribution at $50 \mathrm{~mm}$ and $300 \mathrm{~mm}$ distance from the plasma aperture.

Fig. 3: Calculations with codes ElecNet and Magnet (Infolytica Corp.). ${ }^{12} \mathrm{C}$ (black) and ${ }^{13} \mathrm{C}$ (white) trajectories derived from the calculations in figure 2 are shown for the low-energy beam line of the Woods Hole CFAMS system. Geometric markers are identified in the legend of the figure. (a) Effect of bouncer gap 1 (BG1) on the two masses due to the different bias of the magnet chamber during sequencing; (b) Horizontal and vertical beam profiles showing the larger divergence in the ${ }^{12} \mathrm{C}$ beam; (c) Detail of the horizontal beam profile in the stripper canal indicating larger losses in the lighter mass beam component. 
Figure 1

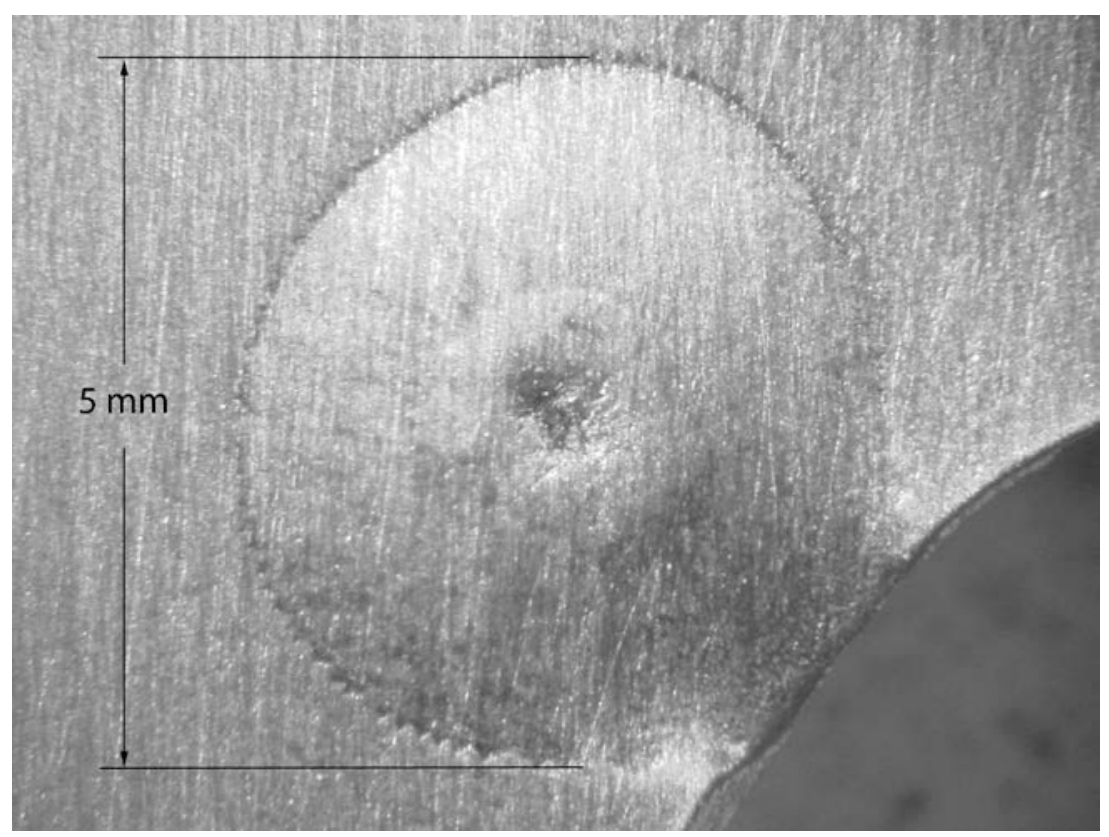

Figure 2
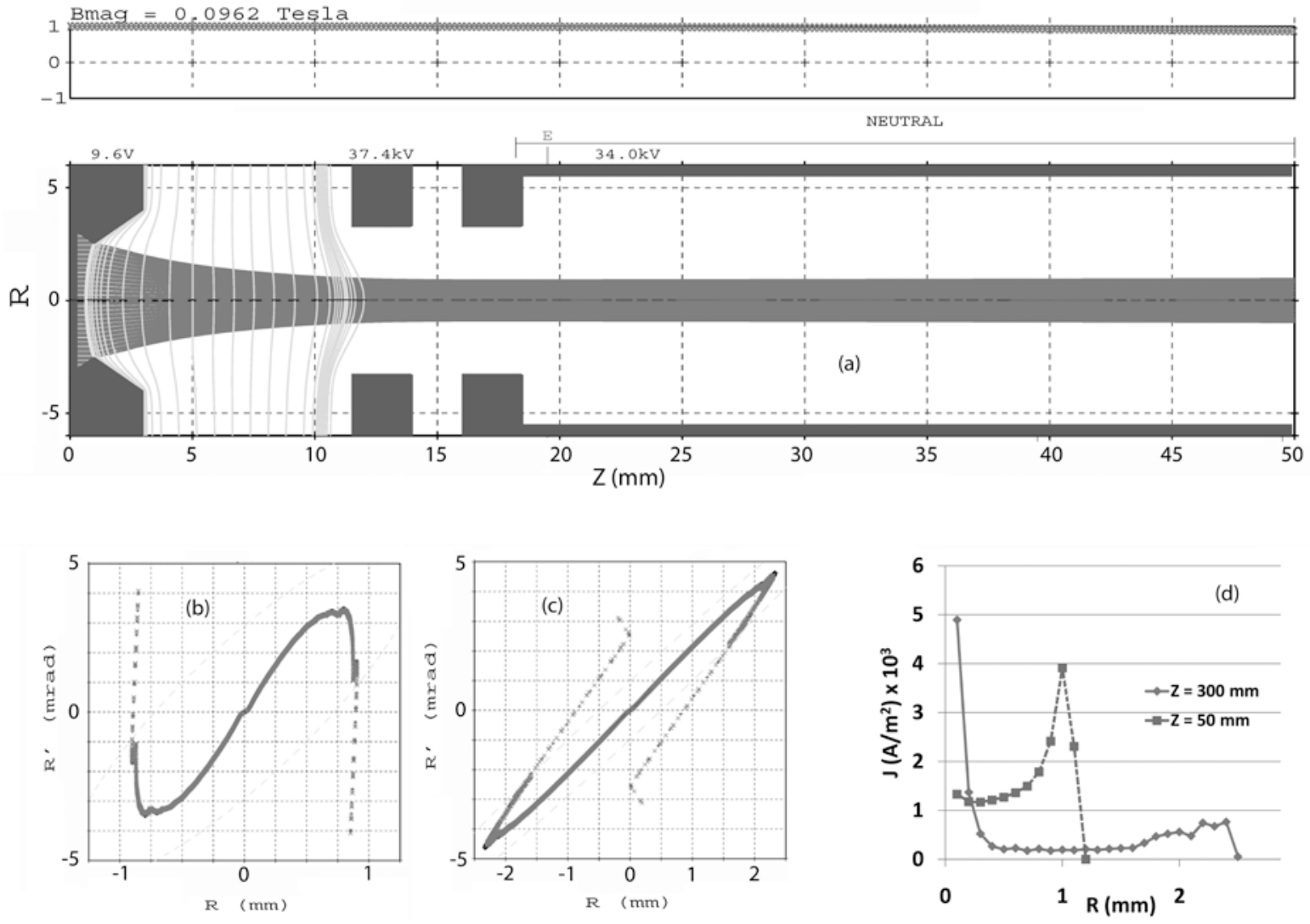
ECAART 10, Athens, Greece - Sep 13-17, 2010: Manuscript 032

Figure 3

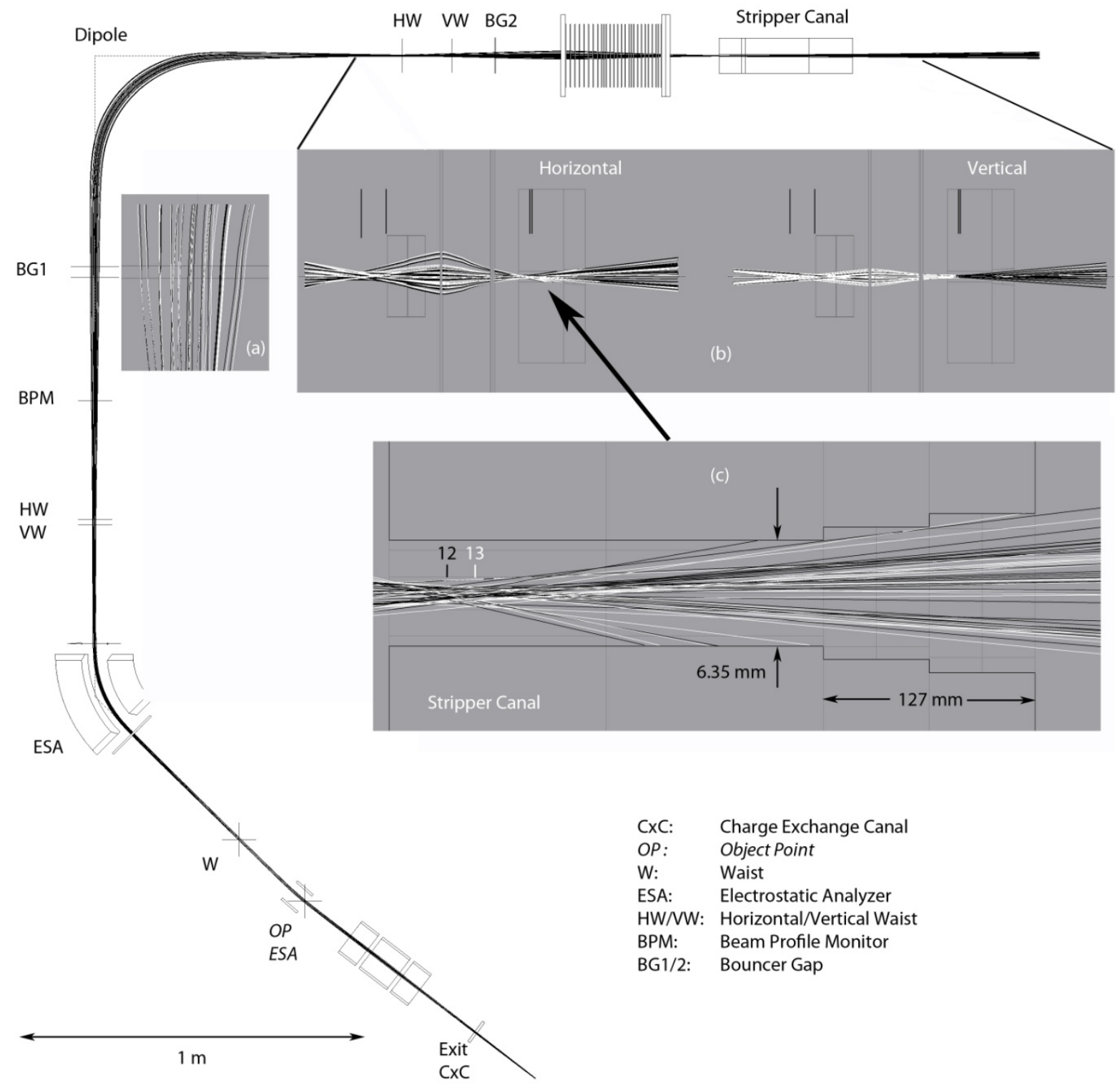

